\title{
Verbinder aus Kunstharzpressholz - Versuche mit ersten Prototypen für Traglasten bis 500 kN
}

Systemverbinder für Holz-Holz-Verbindungen von Nebenträgern an Hauptträger sind zweiteilige Verbinder aus Metall, deren Vorteil sich u. a. im hohen Vorfertigungsgrad auszeichnet. Für die Entwicklung von neuartigen Haupt-NebenträgerSystemverbindern aus Kunstharzpressholz wurden verschiedene Oberflächenbehandlungen untersucht und die jeweiligen Reibbeiwerte zwischen Verbinder und Holz ermittelt. Aufbauend auf diesen Ergebnissen wurden in Vorversuchen Verbindungen mit geneigten Schrauben untersucht. Dabei wurden sowohl die Anordnung als auch die Anzahl und Länge der Schrauben systematisch variiert. Überprüft wurden dabei mögliches Blockscherversagen ganzer Schraubengruppen als auch die wirksame Verbindungsmittelanzahl. Das Prüfprogramm umfasste Versuche mit Lasten in drei Hauptrichtungen für verschiedene Prototypen. Zu Beginn wurden Verbinder für eine Tragfähigkeit in Einschubrichtung von 180 kN geprüft, daraufhin Schwerlastverbinder für Traglasten bis 500 kN. Aus den Versuchen resultierten neue Erkenntnisse für die Bemessung solcher Verbindungen. Das ursprünglich vorgeschlagene Rechenmodell wurde sukzessive angepasst und eine auftretende Moment-Normalkraft-Interaktion in den Schrauben berücksichtigt. Mit einem mittleren Verhältnis aus Versuchsergebnis zu rechnerischer Schätzlast von 1,05 trifft das angepasste Modell die Tragfähigkeit sehr gut. Im direkten Vergleich der charakteristischen Tragfähigkeiten der Prototypen mit handelsüblichen Verbindern schneiden diese sehr gut ab und übertreffen z. T. Verbinder aus Aluminium.

Stichworte Kunstharzpressholz; KPH; Reibung; Systemverbinder; HT-NTVerbinder; MNV-Interaktion

1

Allgemeines

Für Anschlüsse von Nebenträgern an Hauptträger stehen verschiedene Varianten zur Auswahl. Zum einen zimmermannsmäßige Verbindungen wie z.B. der Schwalbenschwanz, zum anderen ingenieurmäßige Verbindungen mit z.B. Winkelverbindern und seit Längerem eben auch Systemverbinder. Deren Vorteil liegt in ihrem hohen Vorfertigungsgrad, da die Verbinderplatten schon im Werk montiert werden und auf der Baustelle nur noch ineinandergesteckt werden müssen. Befestigt werden Systemverbinder hauptsächlich mit geneigten Schrauben, mit welchen die Last aus dem Nebenträger in den Verbinder und dann vom Verbinder in den Hauptträger übertragen wird. Die häufigste Form von handelsüblichen Systemverbindern ähnelt dem Schwalbenschwanz und gibt damit auch die Hauptbeanspruchungsrichtung in Einschubrichtung vor. Auch Kräfte quer zur Einschubrichtung können
Densified veneer wood connectors - tests with first prototypes for loads up to $500 \mathrm{kN}$

System connectors for timber-to-timber secondary beam to main beam connections are two-part connectors made of metal, the advantages of which are, among other things, distinguished by the high degree of prefabrication. For the development of new beam-to-beam connectors, various surface treatments were examined and the respective coefficients of friction were determined. Based on these results, connections with inclined screws were examined in preliminary tests. The arrangement as well as the number and length of screws were systematically varied. Possible block shear failure of entire screw groups, as well as the effective number of fasteners were checked. The test program included tests in the main load directions for various prototypes. First, connectors for a load carrying capacity of $180 \mathrm{kN}$ were tested in the insertion direction, then heavy-duty connectors for loads up to $500 \mathrm{kN}$. New insights for the dimensioning of such connections resulted from the experiments. The originally proposed calculation model was successively adapted and the Moment-Normal force-interaction in the screws was taken into account. With an average ratio of the ultimate test load to the expected capacity of 1.05 , the adapted model predicts the load carrying capacity very well. In a direct comparison of the characteristic load carrying capacities of the prototypes with commercially available connectors, the prototypes perform very well and in some cases outperform conventional connectors made of aluminium.

Keywords densified veneer wood; DVW; friction; system connector; beamto-beam connector; MNV-interaction

übertragen werden, diese sind jedoch um ein Vielfaches geringer. Zur Entwicklung von Haupt-Nebenträger-Verbindern aus Kunstharzpressholz wurde im ersten Teil eines Forschungsvorhabens die Oberflächenbehandlung der Verbinderteile untersucht. Die Ergebnisse wurden ausführlich in [1] vorgestellt. Ein Fazit der Untersuchungen war, dass durch die höhere Reibung zwischen Verbinder und Holzbauteil Tragfähigkeitssteigerungen > 30\% möglich sind. Unabhängig davon wurde in [2] die Tragfähigkeit und Steifigkeit von Holz-Holz-Verbindungen mit geneigten und gekreuzten Schrauben unter Berücksichtigung der Reibung mit FE modelliert. Hierbei wurde bestätigt, dass bei quasistatischen Lasten durch größere Reibbeiwerte Tragfähigkeitssteigerungen von bis zu 30\% möglich sind. Die auf der Reibung beruhende zusätzliche Kraftkomponente wird auch in den Bemessungsvorschlägen für Stahlblech-Holz-Laschenverbindungen in [3] berücksichtigt. Dabei wird der Reibbeiwert $\mu=0,25$ aus 

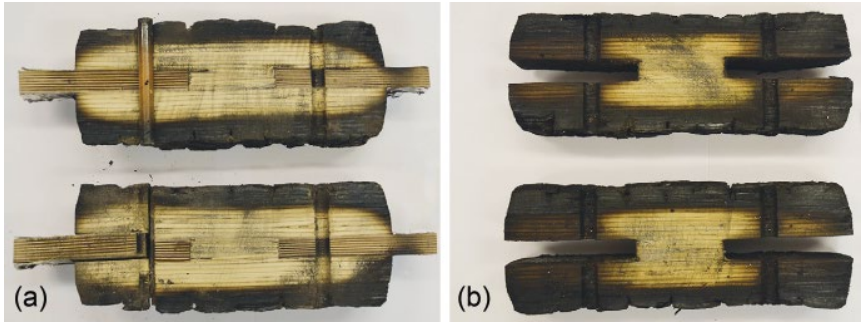

Bild 1 Schnitt durch eine Verbindung mit KPH Knotenplatte (a) und Stahl (b) nach 50 min Brandversuch Longitudinal section of a connection with DVW flitch plate (a) and steel (b) after 50 min of heating

dem Eurocode 5 betrachtet. Als Grundlage für die Bemessungsgleichungen wurden u. a. etwa 600 Stahlblech-HolzZuglaschenverbindungen in Bauteilabmessungen geprüft und der Einfluss der Verbindungsmittelanzahl auf die Tragfähigkeit der Verbindung ermittelt.

Ebenfalls von Bedeutung für Anschlüsse ist der Brandschutz, der mit verschiedenen Ansätzen gewährleistet werden kann. So können der Haupt-/Nebenträger eingefräst und der Verbinder in dieser Nut montiert werden. Der Verbinder ist damit allseitig von Holz umschlossen und vor direkter Beflammung geschützt. Alternativ kann die Verbindung komplett mit Holzwerkstoffplatten verkleidet werden, oder es werden Brandschutzanstriche oder Bänder verwendet. Der große Vorteil von Kunstharzpressholz liegt in den sehr guten brandtechnischen Eigenschaften [4]. Das namensgebende Harz ist ein Phenolharz und wird zur Verleimung auf die Oberfläche der Furniere aufgetragen, wodurch ein Holz-Harz-Verbund aus etwa $70 \%$ Holz und 30\% Harzanteil entsteht. Bei Abbrand verhält sich KPH dementsprechend wie ein Holzwerkstoff und nicht wie Kunststoff. Aufgrund der hohen Rohdichte beträgt die Abbrandrate von KPH nur etwa $0,3 \mathrm{~mm} / \mathrm{min}$. Somit können die Verbinderplatten aus KPH um das jeweils erforderliche Maß größer hergestellt werden und ein Einfräsen oder Verkleiden des An- schlusses ist nicht mehr nötig. Die Wärmeleitfähigkeit von KPH ist ebenfalls sehr gering. In Brandversuchen wurde in [5] der Wärmeeintrag von metallischen und nicht metallischen Verbindern untersucht. Wie in Bild 1 ersichtlich, wird deutlich weniger Wärme in die Verbindung mit Stiften oder Knotenplatten aus KPH eingetragen und der Abbrand ist wesentlich geringer.

\subsection{Prototypverbinder aus Kunstharzpressholz}

In Zusammenarbeit mit der Firma Pitzl Metallbau GmbH in Altheim wurde ein erster Prototyp für einen Verbinder aus KPH entwickelt. Der Fokus lag dabei auf der gefrästen Oberfläche für höhere Tragfähigkeiten und einem kurzen Einführstutzen für eine einfache Montage. Außerdem sollen durch eine mögliche Relativverschiebung der Verbinderplatten rechtwinklig zur Verbinderebene Fertigungstoleranzen der Holzbauteile ausgeglichen werden können. Daraus ergab sich der erste Prototyp v1 in Bild 2a. Für die Oberflächenbehandlung wurde das Pyramidenmuster mit $0,5 \mathrm{~mm}$ tiefen Pyramiden gewählt und für die Abhebesicherung Zylinderschrauben M5 nach DIN 912. Anhand der Versuchsergebnisse und Versagensarten der ersten Verbinderserie wurde ein zweiter geänderter Prototyp v2 hergestellt (Bild 2b). Für die Oberfläche wurde diesmal das Pyramidenmuster mit $1,0 \mathrm{~mm}$ tiefen Pyramiden gewählt und für die Abhebesicherung Zylinderschrauben M6. Außerdem wurden zusätzliche Schrauben für die Montage und zur Verstärkung rechtwinklig zur Verbinderebene angeordnet. Bei beiden Prototypen und allen Versuchen wurden Würth ASSY plus VG $6 \times 200 \mathrm{~mm}$ verwendet.

Außerdem wurde ein erster Prototyp für einen Schwerlastverbinder für Traglasten bis zu $500 \mathrm{kN}$ hergestellt und geprüft (Bild 3). Die Verbinderteile wurden aus $50 \mathrm{~mm}$ dickem, kreuzweise geschichtetem KPH gefräst. In die Oberfläche wurde das Pyramidenmuster mit $1,0 \mathrm{~mm}$ tie-
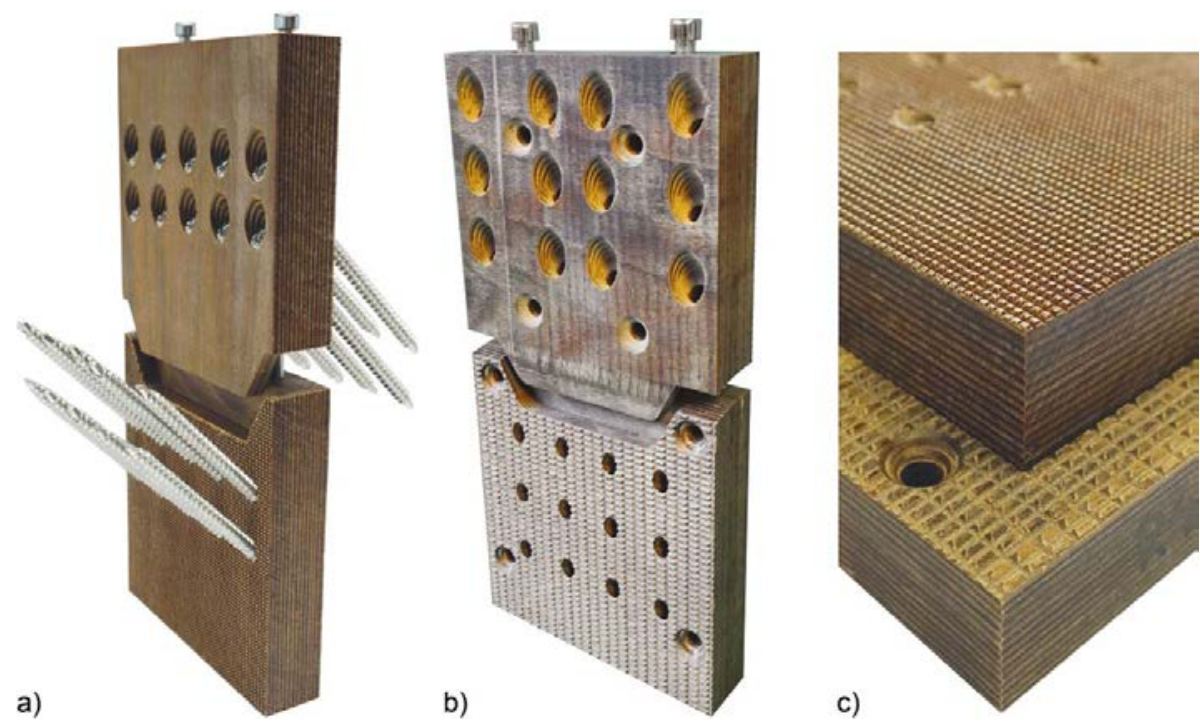

Bild 2 Prototypen für einen Verbinder aus KPH: a) Prototyp v1, b) Prototyp v2, c) gefräste Oberfläche Prototypes for a connector made of DVW: a) prototype v1, b) prototype v2, c) milled surface 

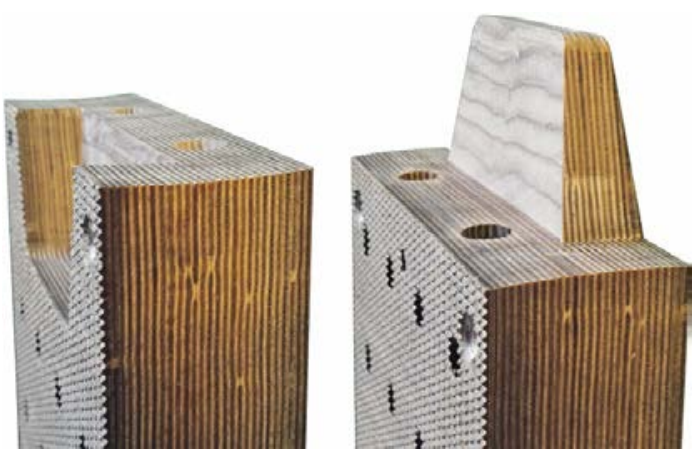

Bild 3

Detailaufnahme eines ersten Prototyps für Traglasten bis 500 kN Close-up of a first prototype for loads up to $500 \mathrm{kN}$

fen Pyramiden gefräst. Die Vollgewindeschrauben für die Kraftübertragung sind um $45^{\circ}$ geneigt. Gewählt wurden hierfür Würth ASSY plus VG $8 \times 300 \mathrm{~mm}$. Insgesamt sind 20 Schrauben pro Verbinderteil in fünf Reihen angeordnet. Als Abhebesicherung dienen zwei Zylinderschrauben M10.

\subsection{Eigenschaften von Schraubenverbindungen in Kunstharzpressholz}

Um die Schätzlast der Prototypverbinder und das mögliche Versagen während der Versuche zu bestimmen, wurden im Vorfeld die Eigenschaften von Holzschrauben in Kunstharzpressholz untersucht. Dazu wurde in Versuchen sowohl die Kopfdurchziehtragfähigkeit als auch die Lochleibungsfestigkeit mit unterschiedlichen Schrauben und Plattendicken ermittelt. Ebenfalls wurde die Herausziehtragfähigkeit verschiedener Schrauben aus KPH bestimmt.

\subsubsection{Kopfdurchziehtragfähigkeit}

Mit den beiden verwendeten Schraubendurchmessern $d=6 \mathrm{~mm}$ und $8 \mathrm{~mm}$ wurde die Kopfdurchziehtragfähigkeit systematisch an vier verschiedenen Plattendicken bestimmt. Die Ergebnisse sind in Tab. 1 angegeben. Ab einer KPH Dicke von $t=15 \mathrm{~mm}$ wird für Schrauben mit $\varnothing 6 \mathrm{~mm}$ die Zugtragfähigkeit maßgebend, bei den Schrauben mit $\varnothing 8 \mathrm{~mm}$ ab $t=25 \mathrm{~mm}$.

\subsubsection{Herausziehtragfähigkeit}

Für die Abhebesicherung bei Belastungen entgegen der Einschubrichtung wurden zwei verschiedene Varianten untersucht. Zum einen Zylinderschrauben mit metrischem Gewinde nach DIN 912, zum anderen Holzschrauben. Bei den Holzschrauben wurde unterschieden zwischen Schrauben vom Typ ASSY plus VG mit Normalgewinde und Schrauben vom Typ ASSY 3.0 TG mit Grobganggewinde. Die Schrauben wurden alle mit Kerndurchmesser vorgebohrt und in die Schmalseite von KPH mit $t=25 \mathrm{~mm}$ eingedreht. Die Ergebnisse sind in Tab. 2 angegeben. Für die Ø6-mm-Schrauben wird ab einer Einbindetiefe von $40 \mathrm{~mm}$ die Zugtragfähigkeit maßgebend, für die Ø8-mm-Schrauben erst ab einer Einbindetiefe von $50 \mathrm{~mm}$. Die max. Einbindetiefe für die metrischen Schrauben ist abhängig von der Gewindelänge nach DIN 912. Bei den Versuchen mit den Holzschrauben kommt es schon bei den kleinsten Einbindetiefen zu Rissen im $\mathrm{KPH}$, sodass Zylinderschrauben als Abhebesicherung gewählt wurden.

Tab. 1 Kopfdurchziehtragfähigkeit in Abhängigkeit von der Plattendicke (Mittelwerte in kN, jeweils $n=3$ )

Head pull-through capacity relative to the panel thickness (mean values in $\mathrm{kN}$, always $n=3$ )

\begin{tabular}{llllll}
\hline Schraubentyp & & Plattendicke & & & \\
& & $\mathbf{6} \mathbf{~ m m}$ & $\mathbf{1 0} \mathbf{~ m m}$ & $\mathbf{1 5} \mathbf{~ m m}$ & $\mathbf{2 5} \mathbf{~ m m}$ \\
\hline $6 \mathrm{~mm}$ & Senkfrästaschenkopf & $4,55 \pm 0,37$ & $8,93 \pm 1,19$ & $15,0 \pm 0,49$ & $15,6 \pm 0,88$ \\
$8 \mathrm{~mm}$ & Senkfrästaschenkopf & $5,16 \pm 0,18$ & $10,6 \pm 0,74$ & $20,7 \pm 0,22$ & $24,5 \pm 0,36$ \\
\hline
\end{tabular}

Tab. 2 Herausziehtragfähigkeit in Abhängigkeit von der Einbindetiefe (Mittelwerte in kN, jeweils $n=5$ ) Withdrawal capacity relative to embedding depth (mean values in $\mathrm{kN}$, always $n=5$ )

\begin{tabular}{|c|c|c|c|c|c|}
\hline \multicolumn{2}{|c|}{ Schraubentyp } & \multicolumn{4}{|c|}{ Einbindetiefe } \\
\hline & & $10 \mathrm{~mm}$ & $20 \mathrm{~mm}$ & $30 \mathrm{~mm}$ & $40 \mathrm{~mm}$ \\
\hline M5 & Metrisches Gewinde & $5,13 \pm 0,58$ & $8,07 \pm 1,44$ & - & - \\
\hline M6 & Metrisches Gewinde & - & - & $13,1 \pm 0,70$ & - \\
\hline M10 & Metrisches Gewinde & - & - & - & $28,2 \pm 1,34$ \\
\hline \multirow[t]{2}{*}{$6 \mathrm{~mm}$} & Normalgewinde & - & $7,62 \pm 0,46$ & $12,9 \pm 1,34$ & $15,4 \pm 0,51$ \\
\hline & Grobganggewinde & - & $8,90 \pm 0,64$ & $13,9 \pm 1,01$ & $15,5 \pm 0,50$ \\
\hline \multirow[t]{2}{*}{$8 \mathrm{~mm}$} & Normalgewinde & - & $8,57 \pm 0,61$ & $14,7 \pm 1,90$ & $21,0 \pm 1,68$ \\
\hline & Grobganggewinde & - & $7,17 \pm 0,27$ & $13,4 \pm 1,61$ & $19,4 \pm 2,86$ \\
\hline
\end{tabular}


Tab. 3 Lochleibungsfestigkeit in Abhängigkeit von der Plattendicke (Mittelwerte in $\mathrm{N} / \mathrm{mm}^{2}$ )

Embedding strength relative to the panel thickness (mean values in $\mathrm{N} / \mathrm{mm}^{2}$ )

\begin{tabular}{lclll}
\hline \multicolumn{2}{l}{ Schraubentyp } & \multicolumn{2}{l}{ Plattendicke } & \\
& & $\mathbf{6} \mathbf{~ m m}$ & $\mathbf{1 0} \mathbf{~ m m}$ & $\mathbf{1 5} \mathbf{~ m m}$ \\
\hline $6 \mathrm{~mm}$ & Vollgewinde & $\begin{array}{l}296 \pm 10 \\
n=10\end{array}$ & $233 \pm 9$ & - \\
& & $n=6$ & \\
$8 \mathrm{~mm}$ & \multirow{2}{*}{ Vollgewinde } & $232 \pm 16$ & $167 \pm 3$ & - \\
& & $n=4$ & $n=7$ & \\
\hline
\end{tabular}

\subsubsection{Lochleibungsfestigkeit}

Zur Bestimmung der Lochleibungsfestigkeit wurden Versuche ebenfalls mit Schrauben $\varnothing 6 \mathrm{~mm}, \varnothing 8 \mathrm{~mm}$ und den Plattendicken $t=6,10,15 \mathrm{~mm}$ durchgeführt. Die Ergebnisse enthält Tab. 3. Es ist ersichtlich, dass die Festigkeit mit zunehmenden Werten der Plattendicke und des Verbindungsmitteldurchmessers abnimmt. Aufgrund der Verformung der Verbindungsmittel bei einer Plattendicke von $15 \mathrm{~mm}$ lässt sich hierfür keine reine Lochleibungsfestigkeit mehr bestimmen. Es wird angenommen, dass sich $\mathrm{KPH}$ in abscherbeanspruchten Verbindungen ähnlich wie Stahlblech verhält.

\section{Vorversuche mit unterschiedlicher Schraubenanzahl, -anordnung und -länge}

Aufbauend auf [1] wurde in weiteren Vorversuchen zuerst die Schraubenanordnung variiert, womit versucht wurde, die Verdrehung der Verbinder zu minimieren. Dazu wurde der Schwerpunkt der Schraubengruppe um den Abstand $e$ so verschoben, dass sich das resultierende Moment aus Einwirkung und Widerstand zu null ergibt. Somit konnte die Verdrehung der Verbinder unterbunden werden, jedoch sank auch die mittlere Tragfähigkeit. Das Versagen trat wie zuvor aufgrund des Erreichens der Zugtragfähigkeit der Schrauben ein.

a)
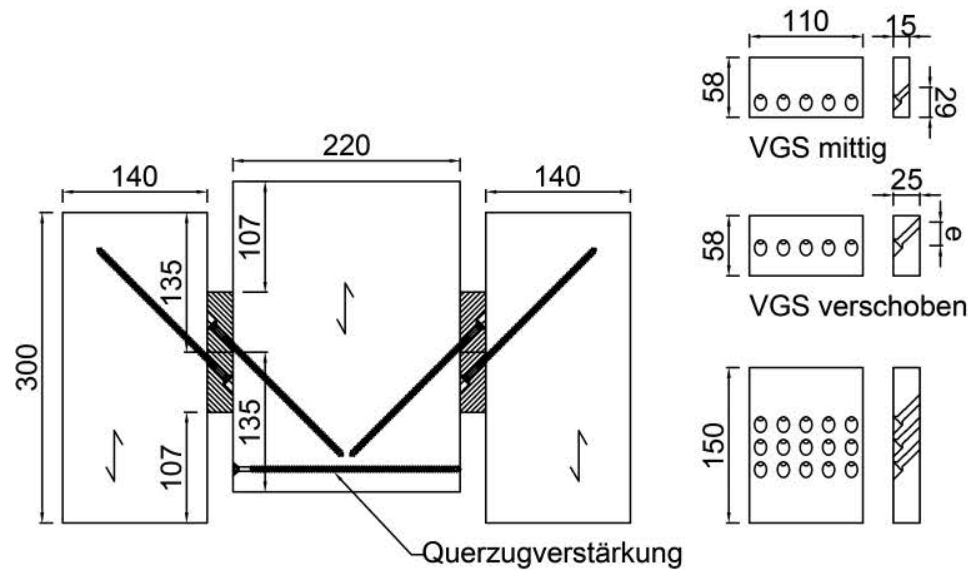

Daraufhin wurde die Anzahl der Schrauben gesteigert, um zum einen zu überprüfen, ob bei der großen Anzahl von sehr dicht angeordneten Schrauben auch Blockscherversagen bei der späteren Bemessung zu berücksichtigen ist. Zum anderen sollte das Rechenmodell für die Hauptbelastungsrichtung $F_{2}$ weiter validiert und die Annahme $n_{\mathrm{ef}}=n$ geprüft werden. Nach Eurocode 5 beträgt bei axial beanspruchten Schraubengruppen die wirksame Anzahl der Schrauben $n_{\mathrm{ef}}=n^{0,9}$. Gewählt wurden Würth ASSY plus VG $6 \times 100 \mathrm{~mm}$, die in drei Reihen à fünf Schrauben angeordnet und um $45^{\circ}$ geneigt waren. Ein Einfluss der großen Anzahl an Schrauben konnte nicht festgestellt werden. Die Versuche versagten alle durch ein Herausziehen der Schrauben aus dem Holz.

Anschließend wurden Versuche mit Schrauben $6 \times 180$ und $6 \times 200 \mathrm{~mm}$ durchgeführt, um ein Herausziehversagen der Schrauben aus dem Holz zu vermeiden. Bei diesen Versuchen trat zum ersten Mal ein Versagen der Verbinderteile auf. Im Nettoquerschnitt in der obersten Schraubenreihe kam es zu einem Druckversagen durch Ausknicken der Furniere. Auffällig zu beobachten war vor dem Druckversagen ein starkes Durchbiegen der Verbinderteile, wodurch das Versagen der Verbinderteile unter Längsdruck begünstigt wurde.

Die Maße der Versuchskörper und der Verbinder sowie die jeweils untersuchte Schraubenanordnung sind in Bild 4 gezeigt. Die wesentlichen Ergebnisse sind in Tab. 4 aufgeführt, die angegebene Rohdichte ist der Mittelwert der verwendeten Seiten- und Mittelhölzer jeder Versuchsreihe.

\section{Versuche mit Prototypverbindern für Traglasten bis zu 180 kN}

\subsection{Versuchsplanung}

Die Versuchsdurchführung umfasste Belastungen in alle Hauptrichtungen: Kraft $F_{1}$ rechtwinklig zur Verbinderebene, Kraft $F_{2}$ in Einschubrichtung, Kraft $F_{45}$ rechtwink-

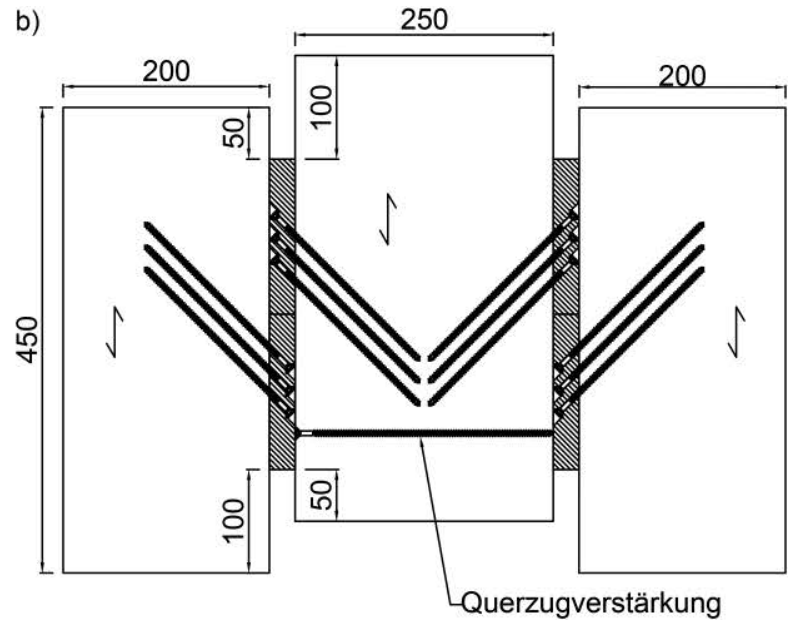

Bild 4 Versuchsaufbau und Schraubenanordnung für Druck-Scher-Versuche mit fünf Schrauben (a) und 15 Schrauben (b) Test setup and screw assembly for push-out tests with five screws (a) and 15 screws (b) 
Tab. 4 Höchstlasten und Steifigkeiten aus den Vorversuchen (Mittelwerte)

Maximum loads and stiffnesses of the preliminary tests (mean values)

\begin{tabular}{|c|c|c|c|c|c|c|}
\hline Oberfläche & Schraubentyp & $\begin{array}{l}\text { Schrauben- } \\
\text { anzahl }\end{array}$ & $\begin{array}{l}\text { Anzahl } \\
\text { Versuche } n\end{array}$ & $\begin{array}{l}\text { Rohdichte } \\
\text { in } \mathbf{~} \mathrm{kg} / \mathrm{m}^{3}\end{array}$ & $\begin{array}{l}F_{2, \max } \\
\text { in } \mathbf{k N}\end{array}$ & $\begin{array}{l}k_{2, \mathrm{~s}} \\
\text { in } \mathrm{kN} / \mathrm{mm}\end{array}$ \\
\hline gefräst kreisförmig & $5 \times 100$ & 5 & 3 & 447 & $49,9 \pm 0,2$ & $14,7 \pm 1,4$ \\
\hline gefräst Pyramiden (1,0 mm) & $5 \times 100$ & 5 & 3 & 440 & $52,9 \pm 4,0$ & $15,6 \pm 0,8$ \\
\hline gefräst Pyramiden $(1,0 \mathrm{~mm})$ verschoben & $5 \times 100$ & 5 & 5 & 455 & $49,1 \pm 3,8$ & $14,1 \pm 2,5$ \\
\hline gefräst Pyramiden (1,5 mm) & $5 \times 100$ & 5 & 3 & 446 & $53,4 \pm 1,3$ & $12,2 \pm 0,7$ \\
\hline gefräst Pyramiden $(1,5 \mathrm{~mm})$ verschoben & $5 \times 100$ & 5 & 5 & 461 & $47,8 \pm 5,4$ & $15,0 \pm 3,2$ \\
\hline gefräst Pyramiden $(0,5 \mathrm{~mm})$ & $6 \times 180$ & 5 & 5 & 445 & $84,5 \pm 5,3$ & $17,8 \pm 1,2$ \\
\hline gefräst kreisförmig & $6 \times 180$ & 5 & 5 & 464 & $80,0 \pm 2,0$ & $17,7 \pm 1,8$ \\
\hline gefräst Pyramiden $(0,5 \mathrm{~mm})$ & $6 \times 100$ & 15 & 5 & 453 & $153 \pm 3,9$ & $34,0 \pm 3,5$ \\
\hline geprägt & $6 \times 100$ & 15 & 5 & 444 & $140 \pm 2,9$ & $42,6 \pm 9,0$ \\
\hline geprägt & $6 \times 200$ & 15 & 3 & 429 & $185 \pm 11$ & $37,4 \pm 3,8$ \\
\hline
\end{tabular}

lig zur Einschubrichtung sowie Versuche mit einer Biegebeanspruchung $M_{2}$ zur Bestimmung der Momententragfähigkeit und der Verdrehung der Verbinder. Der Versuchsablauf erfolgte analog zu DIN EN 26891 mit Entlastungsschleife.

Die Tragfähigkeit $F_{2}$ wurde in zwei verschiedenen Versuchsaufbauten bestimmt. Zuerst wurden Druck-ScherVersuche durchgeführt. Der Aufbau ist sehr kompakt, wie in Bild 5a dargestellt, und eignet sich, um gezielt die Tragfähigkeit eines Verbinders zu ermitteln. Um jedoch das Verhalten und die Tragfähigkeit des Verbinders in einer späteren Einbausituation zu ermitteln, wurden auch Versuche am Gesamtsystem ausgeführt. Dieses besteht aus einem Nebenträger in Bauteilgröße, welcher an beiden Enden jeweils an einem Hauptträger eingehängt wird (Bild 5b). So werden bei der Tragfähigkeit auch Durchbiegungen und Verdrehungen der Bauteile berücksichtigt.

\subsection{Ergebnisse und Diskussion}

Insgesamt wurden mit dem Prototypverbinder v1 zwei Versuche mit Kraft $F_{1}$, neun Versuche mit Kraft $F_{2}$, fünf Versuche mit Kraft $F_{45}$ und zwei Versuche mit Moment $M_{2}$ durchgeführt. Mit dem Prototypverbinder v2 waren es drei Versuche mit Kraft $F_{1}$, zwei Versuche mit Kraft $F_{2}$ und Seitenhölzern aus BSH und zwei zusätzliche Versu- a)
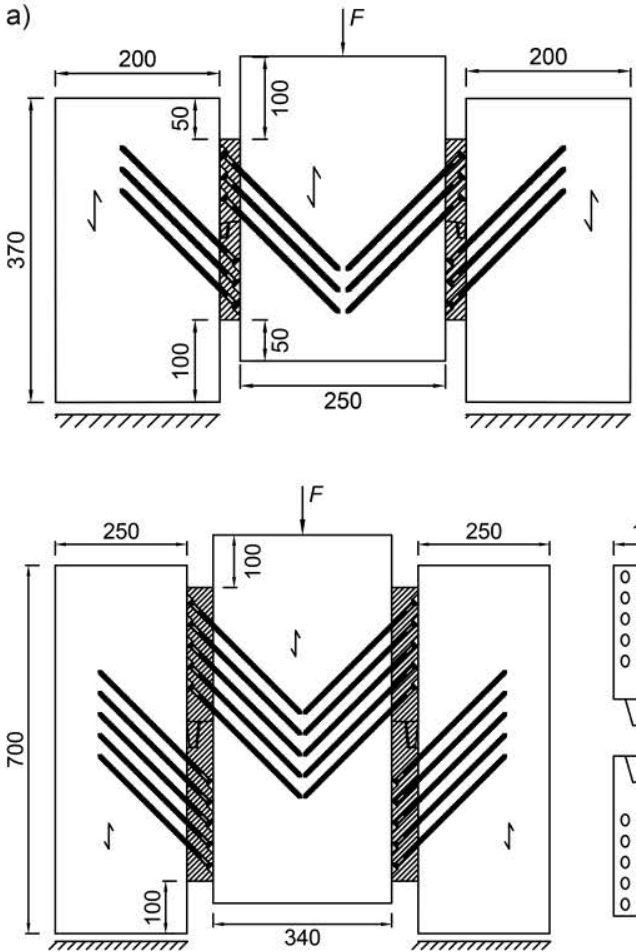

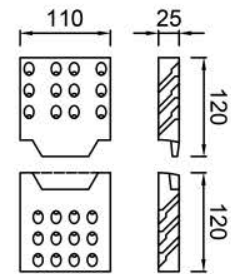

b)

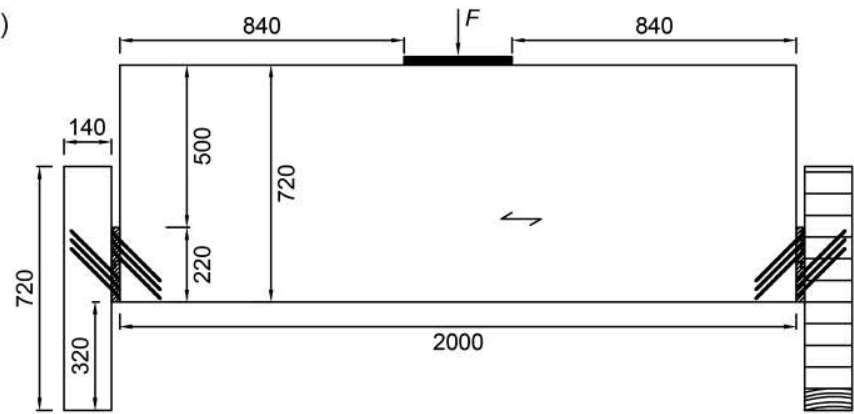

Bild 5 Versuchsaufbau für Druck-Scher-Versuche (a) und HT-NT-Systemversuche (b) Test setup for push-out tests (a) and beam-to-beam system tests (b) 


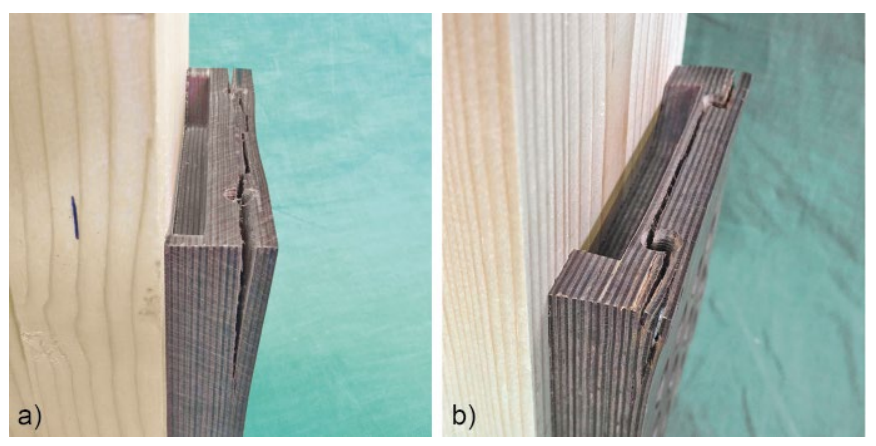

Bild 6 Versagensbilder für die Versuche ohne (a) und mit Montageschrauben (b)

Failure of the connectors without (a) and with assembly screws (b)

che mit BSP, fünf Versuche mit Kraft $F_{45}$ und drei Versuche mit Moment $M_{2}$ sowie vier Versuche am Gesamtsystem. Für alle Versuche wurde BSH GL 24h verwendet, welches bei Normalklima 20/65 gelagert war und eine mittlere Holzfeuchte von $u=11,0 \%$ aufwies. Eine Übersicht über die Ergebnisse zeigt Tab. 5.

\subsubsection{Kraft $F_{1}$ senkrecht zur Verbinderplatte}

Alle Verbinder wurden mit Abhebesicherung eingebaut. Im Mittel lag die Zugkraft im Nebenträger bei $F_{1 \text {,max }}=$ $11,4 \pm 0,4 \mathrm{kN}$ pro Verbinder vom Prototyp v1. Das typische Versagensbild ist in Bild 6a zu sehen. Die Verbinderplatte versagt im Bereich der metrischen Schrauben auf Querzug. Beim zweiten Prototyp v2 wurden diese Bereiche durch die Montageschrauben verstärkt. Die mittlere Zugkraft beim Versagen verdoppelte sich fast auf $F_{1 \text {,max }}=$ $19,4 \pm 0,5 \mathrm{kN}$ pro Verbinder. Das Versagensbild ist analog zur ersten Variante, nur die Rissausbreitung ist geringer (Bild 6b).

\subsubsection{Kraft $F_{2}$ in Einschubrichtung}

Die Verbinder vom Prototyp v1 erreichten im Mittel eine Tragfähigkeit von $F_{2, \max }=151 \pm 5,1 \mathrm{kN}$ und eine Steifigkeit von $k_{2, \mathrm{~s}}=25,0 \pm 4,0 \mathrm{kN} / \mathrm{mm}$. Von acht Versuchskörpern versagten sechs nach Erreichen der Zugtragfähigkeit der Schrauben und zwei nach Erreichen der Drucktragfähigkeit des KPH (Bild 7a). Bei der darauffolgenden Versuchsreihe mit dem zweiten Prototyp v2 konnte eine mittlere Tragfähigkeit von $F_{2, \max }=173 \pm 8,5 \mathrm{kN}$ und eine Steifigkeit von $k_{2, \mathrm{~s}}=33,3 \pm 2,4 \mathrm{kN} / \mathrm{mm}$ ermittelt werden. Aufgrund großer Eindrückungen der Verbinder in die Seitenhölzer aus BSH wurden zwei weitere Versuche mit BSP durchgeführt. Hierbei lag die mittlere Höchstlast bei $F_{2 \text {,max }}=186 \pm 9,1 \mathrm{kN}$, die Steifigkeit konnte durch die Querlagen deutlich erhöht werden auf $k_{2, \mathrm{~s}}=44,3 \pm$ $6,2 \mathrm{kN} / \mathrm{mm}$. Das Versagen war bei allen Versuchen ein Erreichen der Zugtragfähigkeit der Schrauben, nur bei dem letzten Versuch mit BSP kam es zu einem Rollschub-/Querzugversagen in den Querlagen. Die mit dem in [1] vorgestellten Modell bestimmten Schätzlasten lagen über der Tragfähigkeit der Verbindung. Nach den Versuchen waren sehr große Verschiebungen der Verbinder ersichtlich, welche zu Fließgelenken in den geneigten Schrauben führten, wie in Bild $7 \mathrm{~b}$ zu sehen. Daher ist es erforderlich, das Modell entsprechend $\mathrm{zu}$ erweitern. Ebenfalls ersichtlich wurde beim Auftrennen der Prüfkörper ein Verlaufen der Schrauben (Bild 7c). Es konnten jedoch keine beschädigten Schrauben entdeckt werden. Erstaunlich ist, dass bei den in [3] durchgeführten Versuchen keine Biegeverformung sowie kein Verlaufen der Schrauben beobachtet wurde, und das bei ebenfalls sehr großen Tragfähigkeiten und geringen Abständen der Schrauben untereinander.

\subsubsection{Kraft $F_{45}$ senkrecht zur Einschubrichtung}

Die Lasteinleitung erfolgte mittig auf Höhe der Fuge zwischen den beiden Verbinderteilen. Die Verbinder des Prototyps v1 erreichten im Mittel eine Höchstlast von $F_{45 \text {,max }}=20,8 \pm 2,2 \mathrm{kN}$. Das Versagen war eine Kombination aus Abscheren der Wandung, auf welcher der Einführstutzen seitlich aufliegt, sowie einem Scherversagen der Furniere direkt unterhalb der metrischen Schrauben. In Bild 8 sind Versagensbilder von beiden Prototypen zu sehen. Da bei dem Prototyp v2 die senkrechten Montageschrauben zusätzlich als Verstärkung angeordnet waren, konnte eine Steigerung der Tragfähigkeit erreicht werden mit $F_{45, \max }=27,9 \pm 1,5 \mathrm{kN}$.

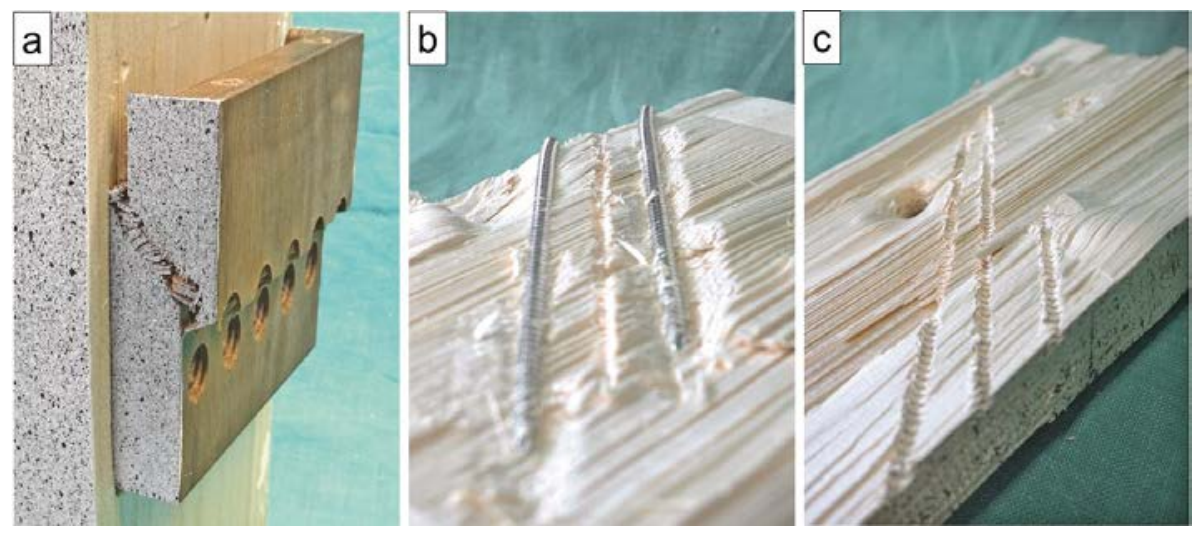

Bild 7 a) Druckversagen der Verbinderplatte, b) deutlich sichtbare Biegeverformung, c) Verlaufen von Schrauben a) Compressive failure of the connector, b) clearly visible bending deformations, c) deviation of the screw axis 


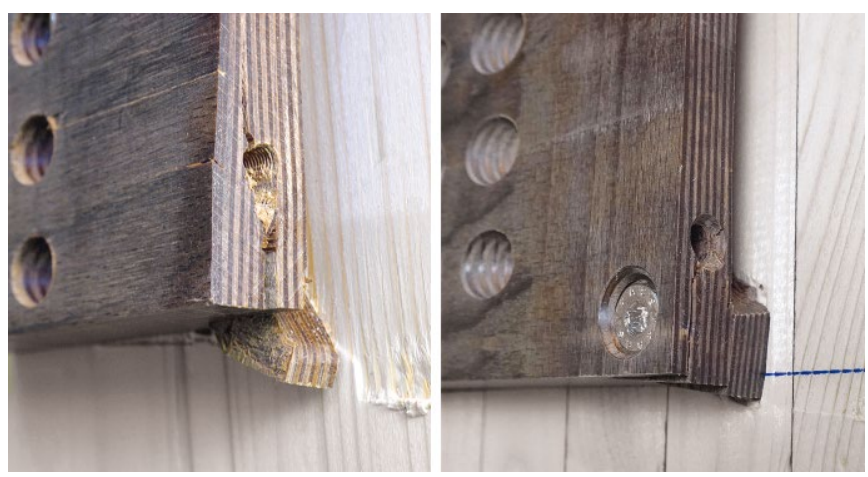

Bild 8 Abscheren der seitlichen Wandung im Bereich des Einführstutzens Shear failure of the side wall beneath the insertion nozzle

\subsubsection{Momententragfähigkeit M2}

Um die Verdrehsteifigkeit der Verbinder zu ermitteln, wurden Versuche mit einer Biegebeanspruchung durchgeführt. Als Versuchsaufbau wurde ein Drei-Punkt-Biegeversuch gewählt, mit den Verbindern in der Mitte an der Stelle des größten Moments. Die Verdrehung berechnet sich als Quotient aus der Relativverschiebung der Oberund Unterkante des Nebenträgers zur Höhe des Nebenträgers (Bild 9a). Im Mittel betrug das max. Moment pro Verbinder vom Prototyp v1 $M_{2, \max }=1,0 \pm 0,03 \mathrm{kNm}$ bei einer Verdrehung von $\varphi_{2, \max }=3,1 \pm 0,3^{\circ}$. Beim Prototyp v2 verdoppelte sich die Momententragfähigkeit auf $M_{2, \max }=2,0 \pm 0,08 \mathrm{kNm}$, während hingegen die Verdrehung etwa gleich blieb mit $\varphi_{2, \max }=2,9 \pm 1,9^{\circ}$. In den Bildern 9b, 9c sind die großen Verformungen gut zu sehen, bei denen dann letztendlich ein Querzugversagen der Verbinderplatte auftrat. In Bild 9c wird die Verstärkung durch die senkrechten Schrauben deutlich.

a)
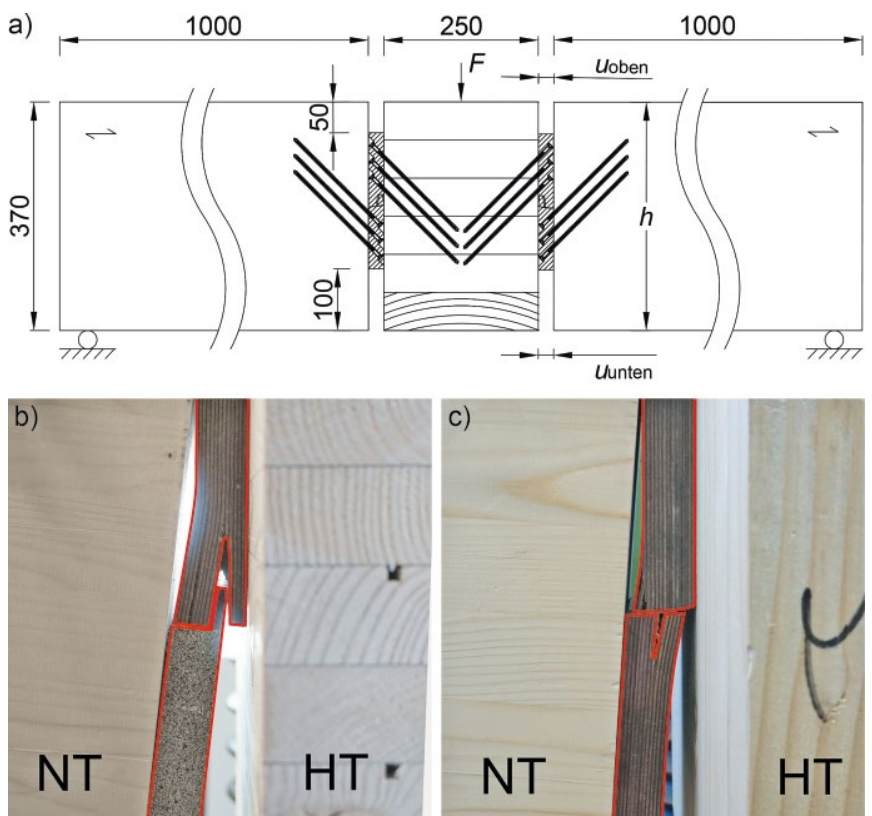

Bild 9 a) Versuchsaufbau zur Ermittlung der Momententragfähigkeit, b) Verformung des Prototyps v1 und c) Verformung des Prototyps v2 unter max. Biegebeanspruchung

a) Test setup for determination of bending load capacity, b) defor mation of the prototype $\mathrm{v} 1$ and c) deformation of the prototype v2 under bending load

\section{$4 \quad$ Versuche mit Prototypverbindern für mittlere Traglasten bis $\mathbf{5 0 0} \mathbf{~ k N}$}

\subsection{Ergebnisse und Diskussion}

Die Versuche beschränkten sich auf drei Versuche in der Kraftrichtung $F_{2}$ mit Seitenhölzern aus Brettschichtholz und einen Versuch mit Brettsperrholz sowie jeweils drei Versuche in Richtung $F_{45}$ und am Gesamtsystem. Für die Seiten- und Mittelhölzer wurde BSH GL 24h verwendet, welches bei Normalklima 20/65 gelagert war und eine mittlere Holzfeuchte von $u=11,4 \%$ aufwies. Eine Übersicht über die Ergebnisse zeigt Tab. 5.

\subsubsection{Kraft $F_{2}$ in Einschubrichtung}

Beim ersten Versuch konnte die Schätzlast nicht erreicht werden, da die beiden Seitenhölzer unterhalb der Verbinderplatten auf Querdruck versagten. Daraufhin wurden die Prüfkörper mit Vollgewindeschrauben $8 \times 160 \mathrm{~mm}$ rechtwinklig zur Faser verstärkt. Bei den folgenden Versuchen kam es zweimal zu einem Druckversagen der Verbinderteile aus KPH (Bild 10a) sowie einmal zum Erreichen der Zugtragfähigkeit der Schrauben. Die mittlere Höchstlast betrug $F_{2, \mathrm{BSH}}=496 \pm 5 \mathrm{kN}$ pro Verbinder und die dazugehörige Steifigkeit $k_{2, \mathrm{~s}}=81 \pm 4 \mathrm{kN} / \mathrm{mm}$. Ein letzter Prüfkörper wurde aus fünflagigem Brettsperrholz hergestellt. Im Versuch konnte nur eine geringfügig höhere Höchstlast erreicht werden mit $F_{2, \mathrm{BSP}}=503 \mathrm{kN}$. Die Steifigkeit der Verbindung konnte jedoch um fast 30\% auf $k_{2, \mathrm{~s}}=105 \mathrm{kN} / \mathrm{mm}$ gesteigert werden. Auch bei diesen Versuchen wurden große Verformungen beobachtet. Nach dem Auftrennen der Versuchskörper war die Verbiegung der Schrägschrauben gut sichtbar (Bild 10b). Dies erklärt die Abweichung der Schätzlast nach Modell von der tatsächlichen Höchstlast. Es muss also auch hier eine Anpassung des Modells erfolgen.

Beim Demontieren eines Verbinders nach dem Versuch wurden beschädigte Schrauben entdeckt. So muss es beim Einschrauben der Schrägschrauben zur Kollision mit einer senkrechten Montageschraube gekommen sein, woraufhin bei zwei Schrauben das Gewinde beschädigt wurde. Die zuerst eingedrehte Montageschraube (Bild 10c) wurde an einer Stelle getroffen. Die später eingedrehte Schrägschraube (Bild 10d) hat ein beschädigtes Gewinde von der Spitze bis zum Kreuzungspunkt der beiden Schrauben. Auf diese Problematik wurde schon in [6] hingewiesen und ein entsprechendes Kegelmodell für den Mindestabstand vorgeschlagen. Dieses wurde hier bei der Wahl der Mindestabstände jedoch nicht angewendet, da die etwa $60 \mathrm{~mm}$ lange Führung der Schrauben durch die Verbinderplatte als ausreichend angesehen wurde. Um zukünftige Kollisionen und Beschädigungen der Schrauben zu verhindern, müssen also entweder die Abstände in Anlehnung an [6] und/oder die Ausrichtung der Schrauben geändert werden. So ist z.B. ein zusätzliches Verschwenken der Schrägschrauben denkbar. 
Tab. 5 Übersicht über die Versuchsergebnisse aller untersuchten Kraftrichtungen der drei Prototypen (Mittelwerte) Summary of the test results of all tested load directions for the three prototypes (mean values)

\begin{tabular}{lllllll}
\hline Kraftrichtung & $\begin{array}{l}\boldsymbol{F}_{\mathbf{1}} \\
\text { in } \mathbf{k N}\end{array}$ & $\begin{array}{l}\boldsymbol{F}_{\mathbf{2}} \\
\text { in } \mathbf{~} \mathbf{N}\end{array}$ & $\begin{array}{l}\boldsymbol{k}_{\mathbf{2}, \mathbf{s}} \\
\text { in } \mathbf{k N} / \mathbf{m m}\end{array}$ & $\begin{array}{l}\boldsymbol{F}_{\mathbf{4 5}} \\
\text { in kN }\end{array}$ & $\begin{array}{l}\boldsymbol{M}_{\mathbf{2}} \\
\text { in } \mathbf{k N m}\end{array}$ & $\begin{array}{l}\boldsymbol{\varphi}_{\mathbf{2}} \\
\text { in }\end{array}$ \\
\hline Prototyp v1 & $11,4 \pm 0,4(n=2)$ & $151 \pm 5,1(n=9)$ & $25,0 \pm 4,0(n=9)$ & $20,8 \pm 2,2(n=5)$ & $1,0 \pm 0,03(n=2)$ & $3,1 \pm 0,3(n=2)$ \\
Prototyp v2 & $19,4 \pm 0,5(n=3)$ & $173 \pm 8,5(n=2)$ & $33,3 \pm 2,4(n=2)$ & $27,9 \pm 1,5(n=5)$ & $2,0 \pm 0,08(n=3)$ & $2,9 \pm 1,9(n=3)$ \\
& & $186 \pm 9,1(n=2)^{1)}$ & $44,3 \pm 6,2(n=2)^{1)}$ & & & \\
Prototyp 500 kN & & $496 \pm 4,9(n=3)$ & $80,1 \pm 4,3(n=3)$ & $83,9 \pm 4,8(n=3)$ & \\
& & $503(n=1)^{1)}$ & $105(n=1)^{1)}$ & & & \\
\hline
\end{tabular}

1) Seiten-/Mittelhölzer aus BSP
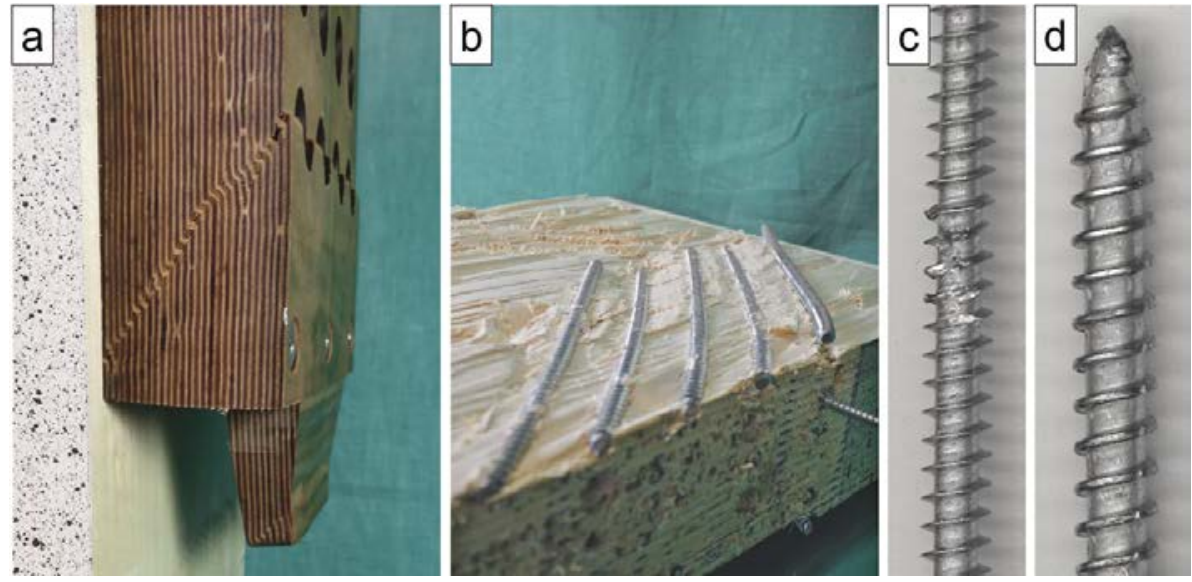

Bild 10 a) Druckversagen des Verbinders aus KPH im Nettoquerschnitt und am Einführstutzen, b) Biegeverformung der Schrägschrauben, c) punktuell geschädigte Montageschraube, d) geschädigtes Gewinde einer Schrägschraube

a) Compressive failure of the DVW in the net area and the insert nozzle, b) bending deformation of the inclined screws, c) local damage of an assembly screw, d) damaged thread of an inclined screw

\subsubsection{Kraft $F_{45}$ senkrecht zur Einschubrichtung}

Bei den Versuchen wurde im Mittel eine Höchstlast von $F_{45, \text { max }}=83,9 \pm 4,8 \mathrm{kN}$ erreicht. Das Versagen ist auf ein Abscheren der seitlichen Wandung zurückzuführen, auf welcher der Einführstutzen aufliegt. Die senkrechten Montageschrauben verstärken diesen Bereich der Verbinderplatte und es kam zu Lochleibungsversagen und der Bildung von zwei Fließgelenken.

\section{Großmaßstäbliche Hauptträger-Nebenträger- Systemversuche}

Um das Verhalten der Verbinder am Gesamtsystem zu untersuchen, wurden großformatige Haupt-NebenträgerVersuche durchgeführt. Der Versuchsaufbau ist in Bild $5 \mathrm{~b}$ zu sehen. Für die Haupt-/Nebenträger wurde BSH GL $24 \mathrm{~h}$ verwendet, welches bei Normalklima 20/65 gelagert war und eine mittlere Holzfeuchte von $u=11,5 \%$ aufwies. Die Holzbauteile wurden wie folgt verstärkt: eine Querdruckverstärkung im Lasteinleitungsbereich und am Auflager sowie eine Querzugverstärkung in direkter Nähe der Verbinder. Außerdem wurden hier bei beiden untersuchten Verbindern die Hauptträger unter den Verbinderplatten mit Vollgewindeschrauben verstärkt, um ein vorzeitiges Querdruckversagen auszuschließen. Hierfür wurden die Vollgewindeschrauben um 2,5 mm im Hauptträger versenkt, sodass sich das gefräste Pyramidenmuster trotzdem noch in das Nadelholz eindrücken konnte. Der Versuchsablauf erfolgte wie zuvor mit Entlastungsschleife.

\subsection{Ergebnisse und Diskussion}

Nach den Versuchen wurden die Höchstlast pro Verbinder sowie die Steifigkeit ausgewertet. Eine Übersicht ist in Tab. 6 gegeben, die angegebene Rohdichte ist der Mittelwert der verwendeten Seiten- und Mittelhölzer jeder Versuchsreihe. Bei den Versuchen mit dem Prototyp v2 drückten sich die Verbinderplatten am Hauptträger trotz der Querdruckverstärkung sehr stark ins Holz ein, sodass es so zum Versagen kam (Bild 11a). Nur beim letzten Versuch kam es zu einem Herausziehversagen der Schrauben aus dem Nebenträger. Dennoch stimmen die mittlere Höchstlast sowie die Steifigkeit gut mit den Ergebnissen aus den Druck-Scher-Versuchen aus Abschn. 3.2.2 überein (Bild 12). Das aufgetretene Querdruckversagen des Hauptträgers lässt sich relativ einfach verhindern: mit der zu Beginn angesprochenen Vergrößerung der Verbinderplatten um die entsprechende Abbrandtiefe vergrößert sich auch automatisch die beim Querdrucknachweis zur Verfügung stehende effektive Fläche. 


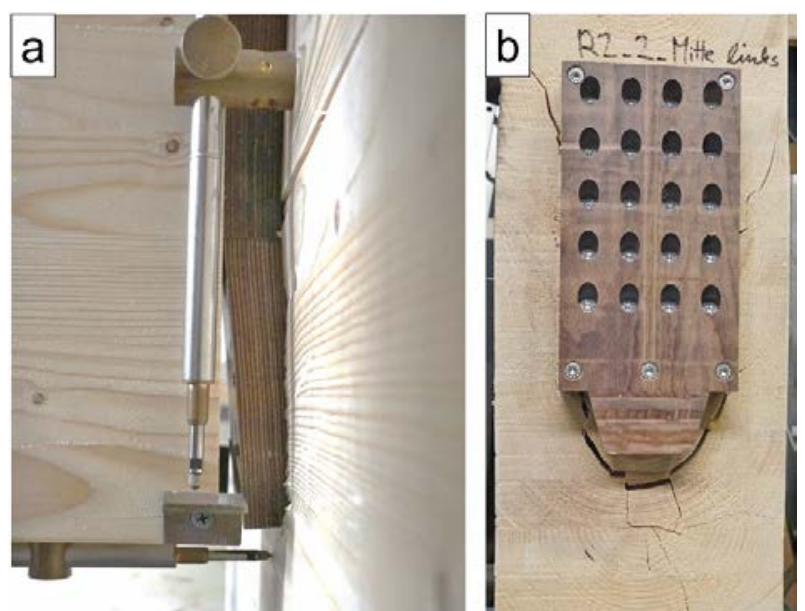

Bild 11 HT-NT-Systemversuche: a) Querdruckversagen am Hauptträger, b) Querzugversagen am Nebenträger

Beam-to-beam system tests: a) compressive failure perpendicular to the grain in the main beam, b) tensile failure perpendicular to the grain in the secondary beam

Tab. 6 Mittlere Höchstlasten aus den Versuchen am Gesamtsystem für beide geprüften Prototypen

Mean ultimate loads for the tests on the entire system for both tested prototypes

\begin{tabular}{lcccc}
\hline Verbinder & $\boldsymbol{F}_{\mathbf{2}}$ in $\mathbf{k N}$ & $\begin{array}{l}\boldsymbol{k}_{\mathbf{2}, \mathbf{s}} \text { in } \\
\mathbf{k N} / \mathbf{m m}\end{array}$ & $\begin{array}{l}\text { Anzahl } \\
\text { Versuche }\end{array}$ & $\begin{array}{l}\text { Rohdichte } \\
\text { in } \mathbf{k g} / \mathbf{m}^{\mathbf{3}}\end{array}$ \\
\hline Prototyp v2 & $172 \pm 7,0$ & $31,1 \pm 3,2$ & 4 & 452 \\
Prototyp 500 kN & $394 \pm 33$ & $71,6 \pm 2,6$ & 3 & 433 \\
\hline
\end{tabular}

Bei den Versuchen mit dem Prototyp $500 \mathrm{kN}$ konnte nicht das volle Potenzial der Verbinder ausgeschöpft und die Ergebnisse der Druck-Scher-Versuche aus Abschn. 4.1.1 reproduziert werden. Hierbei kam es zu Querzugversagen am Hirnholzende im Nebenträger, und zwar im Bereich der geneigten Schrauben (Bild 11b). Bei einem letzten Versuch wurde hier zusätzlich eine Querzugverstärkung angeordnet. Dadurch konnte die Tragfähigkeit zwar nochmal etwas gesteigert werden, aber dennoch blieb der Prototyp hinter den Erwartungen aus den Druck-Scher-Versuchen zurück. Wie in Bild 12 zu sehen, kommt es schon bei kleinen Verformungen zu Unterschieden.

\section{Rechenmodell mit Berücksichtigung von Moment- Normalkraft-Interaktion}

In [1] wurde das Modell zu Berechnung der Tragfähigkeit in Einschubrichtung $F_{\mathrm{V} \text {,exp }}$ einer Verbindung mit um den Winkel $\alpha$ geneigten Schrauben vorgestellt (Gl. (1)). Eingangswerte bei dem Modell sind u.a. das Minimum aus der Herausziehtragfähigkeit und der Zugtragfähigkeit der Schrauben sowie der max. statische Reibbeiwert in der Scherfuge.

$$
F_{\mathrm{V}, \exp }=n_{\mathrm{ef}} \cdot F_{\mathrm{ax}} \cdot(\cos \alpha+\mu \sin \alpha)
$$

Für die ersten Versuche mit kurzen Schraubenlängen und fünf Schrauben stimmten die berechneten Tragfähigkeiten nach Gl. (1) sehr gut mit den Versuchsergebnissen überein. Das Verhältnis von Versuchsergebnis in Einschubrichtung zu Modell liegt im Mittel bei 1,0 $\pm 0,1$ $(n=126)$. Für die Versuche mit 15 Schrauben $6 \times 100 \mathrm{~mm}$ ist das Verhältnis im Mittel mit 1,1 $\pm 0(n=40)$ größer als 1,0. Bei Versuchen mit längeren Schrauben, die so dimensioniert waren, dass das Erreichen der Zugtragfähigkeit der Schrauben maßgebend wird, überschätzt das Modell jedoch die Tragfähigkeit, wie in Bild 13 an den grünen Punkten zu sehen ist.

Zuerst wurde der Reibbeiwert näher betrachtet. Der Reibbeiwert berechnete sich in [1] jeweils als das max. Verhältnis von Reibungskraft zu Normalkraft und somit bei sehr geringen Verformungen. Die Druck-Scher-Versuche haben jedoch gezeigt, dass hier die max. Kraft bei deutlich größeren Relativverschiebungen eintritt, als sie es bei der Auswertung der Reibbeiwerte waren. Daher wurden die Reibungsversuche neu ausgewertet und der Reibbeiwert bei derselben Verformung ausgewertet, wie sie auch in den Druck-Scher-Versuchen auftrat. Dieser angepasste Reibbeiwert lag zwischen 80 und $90 \%$ des statischen Reibbeiwerts und somit noch deutlich über dem Gleitreibungskoeffizienten. Die mit dem angepassten Reibbeiwert ermittelten rechnerischen Tragfähigkeiten kommen bereits deutlich näher an die Versuchsergebnisse heran, überschätzen jedoch weiterhin die Tragfähigkeit, v. a. in den Versuchen mit den zuvor vorgestellten Prototypen (blaue Punkte in Bild 13).
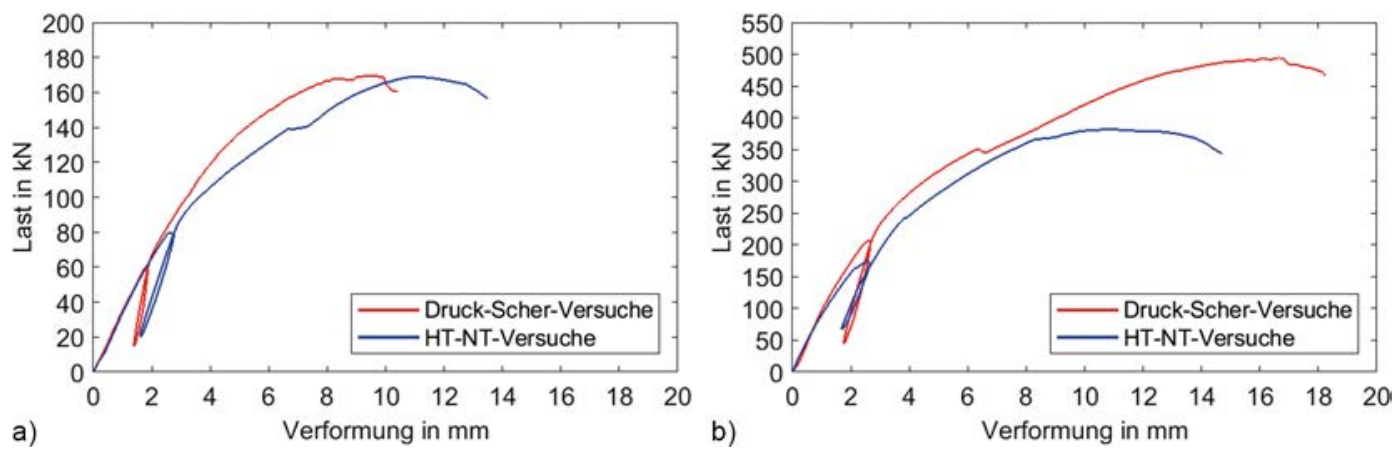

Bild 12 Kraft-Verformungsdiagramme (gemittelt) für Druck-Scher-Versuche und HT-NT-Versuche für den Prototyp v2 (a) und den Prototyp 500 kN (b) Load displacement diagrams (averaged) for the push-out tests and the beam-to-beam tests for the prototype v2 (a) and the prototype 500 kN (b) 


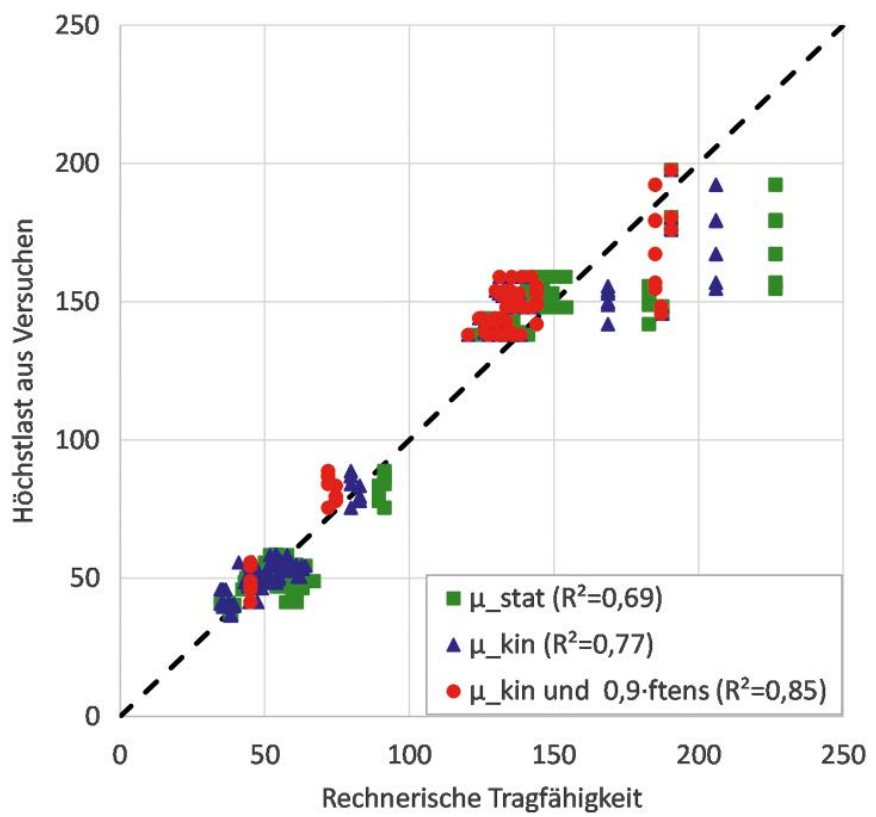

Bild 13 Vergleich des Modells mit den Versuchsergebnissen Comparison of the model and the test results

Bei genauerer Betrachtung dieser Versuche hat sich gezeigt, dass die Verbinder sehr große Relativverschiebungen $\mathrm{zu}$ den Holzbauteilen aufweisen und es zu Fließgelenken in den Vollgewindeschrauben kommt, wie in den Bildern 7, $10 \mathrm{zu}$ sehen ist. Um zu überprüfen, ob eine Momenten-Normalkraft-Interaktion die Schraubentragfähigkeit reduziert, eignet sich Gl. (2), die in [7] vorgestellt wurde. Dabei wurde das Versagen von abscherbeanspruchten Verbindungen aufgrund des Abscherens des Verbindungsmittels untersucht und ein analytisches Modell hergeleitet. Solange die Interaktionsbeziehung nach Gl. (2) eingehalten ist, können die Bemessungsgleichungen nach Eurocode 5 verwendet werden. Wird die Bedingung der Gl. (2) nicht erfüllt, müssen die Eigenschaften des Verbindungsmittels entsprechend verringert werden.

$$
\frac{M}{M_{\mathrm{y}}}+\left(\frac{N}{f_{\text {tens }}}+\frac{V}{f_{\text {shear }}}\right)^{2}=1
$$

Mit der Normalkraft $N$ in der Schraube kann man über die Flächenaufteilung des Kreisquerschnitts der Schraube das zeitgleich wirkende Moment $M$ berechnen. Setzt man diese Werte zusammen mit dem Fließmoment $M_{\mathrm{y}}$ sowie der Zugtragfähigkeit $f_{\text {tens }}$ in Gl. (2) ein, erhält man für die Versuche mit den Prototypen Ausnutzungsgrade $>1,0$. Die Querkraft wird dabei vernachlässigt. Zum einen werden die Schrauben aufgrund der geneigten Anordnung fast ausschließlich auf Zug beansprucht, zum anderen liegen die Fließgelenke, aufgrund der Einspannwirkung im KPH, sehr nahe an der Scherfuge. Somit ist auch nach Gl. (2) rechnerisch nachgewiesen, dass nicht der gesamte Querschnitt der Schraube für die Aufnahme der Zugkraft zur Verfügung steht. Daher wird vorgeschlagen, die Zugtragfähigkeit der Schrauben abzumindern. Wertet man das Verhältnis von tatsächlich vorhandener Normalkraft $N$ zu max. möglicher Normalkraft $F_{\text {tens }}$ aus, resul- tiert ein Mittelwert von 0,9. In Bild 13 sind die neu berechneten Tragfähigkeiten mit der reduzierten Zugtragfähigkeit in Rot eingezeichnet. Der durchschnittliche Verhältniswert von Versuchsergebnissen zu rechnerischer Tragfähigkeit beträgt 1,04 $\pm 0,1(n=183)$ und auch das Bestimmtheitsmaß ist mit $R^{2}=0,85$ deutlich gestiegen.

Zusätzlich müssen weitere Versagensmechanismen berücksichtigt werden. Zum einen muss die Druckfestigkeit in Plattenebene der Verbinder aus KPH ausreichend hoch sein, um ein Versagen im Nettoquerschnitt zu vermeiden. Zum anderen haben die Versuche mit dem Schwerlastprototyp gezeigt, dass bei dieser Verbindergeometrie auch ein mögliches Querdruckversagen im Hauptträger überprüft werden muss. Aufgrund der großen Anzahl an Schrauben auf kleiner Fläche entstehen lokal unter den Verbinderplatten sehr große Spannungen rechtwinklig zur Faser. Somit ergibt sich die Gesamttragfähigkeit der Verbindung mit geneigten Schrauben aus dem Minimum aus Gl. (3). Die ersten beiden Zeilen entstammen dem Modell aus [1], in welches zum einen die Herausziehtragfähigkeit und zum anderen die abgeminderte Zugtragfähigkeit eingesetzt wird. Zeile 3 berücksichtigt das Versagen des Verbinders auf Längsdruck und Zeile 4 das Versagen des Hauptträgers auf Querdruck, wie die zuvor vorgestellten Versuche gezeigt haben.

$$
F_{\mathrm{V}, \text { mean }}=\min \left\{\begin{array}{l}
n \cdot F_{\mathrm{ax}} \cdot(\mu \cdot \sin \alpha+\cos \alpha) \\
n \cdot 0,9 \cdot F_{\mathrm{tens}} \cdot(\mu \cdot \sin \alpha+\cos \alpha) \\
A_{\mathrm{netto}, \mathrm{KPH}} \cdot f_{\mathrm{c}, 0, \mathrm{KPH}} \\
A_{\mathrm{c}, 90, \mathrm{HT}} \cdot f_{\mathrm{c}, 90, \mathrm{HT}} \cdot k_{\mathrm{c}, 90, \mathrm{HT}} \cdot\left(\mu+\frac{1}{\tan \alpha}\right)
\end{array}\right.
$$

mit
$F_{\mathrm{ax}}$
$F_{\text {tens }}$
$\mu$
$\alpha$

$A_{\text {netto, } \mathrm{KPH}}$

$f_{\mathrm{c}, 0, \mathrm{KPH}}$

$A_{\mathrm{c}, 90, \mathrm{HT}}$

$f_{\mathrm{c}, 90, \mathrm{HT}}$

$k_{\mathrm{c}, 90, \mathrm{HT}}$
Verbindungsmittelanzahl

Herausziehtragfähigkeit der Schraube Zugtragfähigkeit der Schraube Reibbeiwert

Einschraubwinkel der geneigten Schrauben gegenüber der Verbinderebene

Nettoquerschnittsfläche des Verbinders

Längsdruckfestigkeit des Verbinders querdruckbeanspruchte Fläche unter der Verbinderplatte am Hauptträger Querdruckfestigkeit des Hauptträgers Querdruckbeiwert

\section{7}

Fazit

Die Versuche haben gezeigt, dass mit Kunstharzpressholz leistungsstarke Verbinder hergestellt werden können. Über die neuartige Form lassen sich auf kleinstem Raum viele Schrauben anordnen und somit hohe Tragfähigkeiten erzielen. Vergleicht man die charakteristischen Tragfähigkeiten von aktuell erhältlichen Hauptträger-Nebenträger-Verbindern mit ähnlichen Dimensionen, dann sind mit Verbindern mit aufgerauter Oberfläche und geneigt 
Tab. 7 Vergleich der charakteristischen Tragfähigkeiten mit handelsüblichen HT-NT-Verbindern

Comparison of characteristic load-carrying capacities with customary beam-to-beam connectors

\begin{tabular}{|c|c|c|c|c|c|c|c|}
\hline Verbinder & $\begin{array}{l}\text { Breite } \\
\text { in } \mathbf{~ m m}\end{array}$ & $\begin{array}{l}\text { Höhe } \\
\text { in mm }\end{array}$ & $\begin{array}{l}\text { Dicke } \\
\text { in } \mathbf{~ m m}\end{array}$ & $\begin{array}{l}\text { Schraubentyp } \\
\text { in } \mathbf{m m}\end{array}$ & Schraubenanzahl & $\begin{array}{l}R_{2, \mathbf{k}}^{1)} \\
\text { in } \mathbf{k N}\end{array}$ & $\begin{array}{l}\text { Kraft pro Schraube } \\
\text { in } \mathbf{k N}\end{array}$ \\
\hline Prototyp v2 & 110 & 220 & 25 & $6 \times 200$ & 24 & $154^{2)}$ & 6,4 \\
\hline Pitzl HVP 88425 & 120 & 250 & $15+15$ & $8 \times 200$ & 20 & 93,3 & 4,7 \\
\hline Sherpa XL 55 & 120 & 250 & $16+16$ & $8 \times 200$ & 18 & 81,9 & 4,6 \\
\hline Prototyp $500 \mathrm{kN}$ & 140 & 558 & 50 & $8 \times 300$ & 40 & $330^{2)}$ & 8,3 \\
\hline Pitzl HVP 88555 & 140 & 550 & $15+15$ & $8 \times 300$ & 56 & 395 & 7,1 \\
\hline Sherpa XXL 280 & 140 & 570 & $16+16$ & $8 \times 200^{3)}$ & 54 & 349 & 6,5 \\
\hline
\end{tabular}

1) für BSH GL 24h

2) $5 \%$-Quantil nach DIN EN 14358 mit $k_{s}(n)=2,1$

${ }^{3)}$ max. zulässige Schraubenlänge nach ETA-12/0067

eingedrehten Schrauben deutliche Tragfähigkeitssteigerungen zu verzeichnen. Dies wird besonders anschaulich, wenn die max. übertragbare Kraft pro Schraube berechnet wird (Tab. 7). Hieraus ergeben sich Tragfähigkeitssteigerungen in Einschubrichtung der Verbinder von 36\% für den Prototyp v2 und 17\% für den Prototyp $500 \mathrm{kN}$. Weiterhin hat die Auswertung der Druck-Scher-Versuche mit den Seiten-/Mittelhölzern aus Brettsperrholz gezeigt, dass die Steifigkeit von Verbindungen deutlich gesteigert werden kann, wenn Nebenträger aus BSP verwendet werden.

\section{Literatur}

[1] Aurand, S.; Blaß, H. J. (2020) Verbinder aus Kunstharzpressholz mit veränderter Oberfläche zur Erhöhung der Reibung in der Scherfuge in: Bautechnik 97, H. S1, S. 44-55.

[2] Azinović, B.; Frese, M. (2020) FE modelling of timber connections with inclined and cross-wise arranged screws new findings on testing the shear stiffness in: Görlacher, R. [ed.] Proceedings of the INTER Meeting 53. Online, 17.-19. Aug. 2020.

[3] Krenn, H. (2018) Die Stahlblech-Holz-Laschenverbindung mit schrägen Schrauben. Monographic Series TU Graz/ Timber Engineering \& Technology, Bd. 7. Graz: Verlag der Technischen Universität Graz.

[4] Gilka-Bötzow, A.; Heiduschke, A.; Haller, P. (2011) Zur Abbrandrate von Holz in Abhängigkeit der Rohdichte in: European Journal of Wood and Wood Products 69, no. 1, pp. 159-162.

\section{Dank}

Die hier vorgestellten Ergebnisse sind im Rahmen des FuE-Kooperationsprojekts „Entwicklung hochleistungsfähiger und brandsicherer Holzverbinder aus Kunstharzpressholz (KPH-Verbinder)" in Zusammenarbeit mit der Fa. Pitzl Metallbau GmbH in Altheim entstanden. Gefördert wurde das Projekt von der Arbeitsgemeinschaft industrieller Forschungsgemeinschaften AiF unter dem Förderkennzeichen FKZ ZF4250805WZ8.

[5] Brandon, D.; Maluk, C.; Ansell, M. P.; Harris, R.; Walker, P.; Bisby, L.; Bregulla, J. (2015) Fire performance of metalfree timber connections in: Proceedings of the Institution of Civil Engineers - Construction Materials 168, no. 4, pp. $173-186$.

[6] Frese, M.; Jordan, M. (2018) Deviations between planned and actual position of wood screws - consequences for minimum spacing in: Görlacher, R. [ed.] Proceedings of the INTER Meeting 51. Tallinn, Estonia, 13.-16. Aug. 2018.

[7] Blaß, H. J. (2018) Moment-Normalkraft-Querkraft Interaktion in stiftförmigen Verbindungsmitteln von StahlblechHolz-Verbindungen in: Görlacher, R.; Sandhaas, C. [Hrsg.] Karlsruher Tage 2018 Holzbau. Forschung für die Praxis. Karlsruhe, 4./5. Okt. 2018.

\footnotetext{
Autoren

Simon Aurand, M.Sc. (Korrespondenzautor)

simon.aurand@kit.edu

Karlsruher Institut für Technologie (KIT)

Holzbau und Baukonstruktionen

R.-Baumeister-Platz 1

76131 Karlsruhe

Prof. Dr.-Ing. Hans Joachim Blaß

hans.blass@kit.edu

Karlsruher Institut für Technologie (KIT)

Holzbau und Baukonstruktionen

R.-Baumeister-Platz 1

76131 Karlsruhe
} 
Karlsruher Institut für Technologie

\section{Repository KITopen}

Dies ist ein Postprint/begutachtetes Manuskript.

Empfohlene Zitierung:

Aurand, S.; Blaß, H. J. Verbinder aus Kunstharzpressholz - Versuche mit ersten Prototypen für Traglasten bis 500 $\mathrm{kN}$.

2021. Bautechnik. doi:10.1002/bate.202000103

DOI: $\underline{10.5445 / / R / 1000129082}$

Zitierung der Originalveröffentlichung:

Aurand, S.; Blaß, H. J.

Verbinder aus Kunstharzpressholz - Versuche mit ersten Prototypen für Traglasten bis 500 $\mathrm{kN}$.

2021. Bautechnik. doi:10.1002/bate.202000103 